\title{
MISTREATMENT ALGORITHMIC PROGRAM TO FIND FREQUENT SEQUENTIAL PATTERNS WHILE NOT ORDERING THE STUMPS SUB SEQUENCES
}

\author{
K. Subramanian ${ }^{1}$, S.Surya ${ }^{2}$ \\ ${ }^{1}$ Assistant Professor, H.H The Rajah's College, Pudukkottai, Tamil Nadu, India. \\ ${ }^{2}$ Ph.D. Research Scholar, J.J. College of Arts and Science, Pudukkottai, Tamil Nadu, India
}

\begin{abstract}
Information-Mining is that a method otherwise an association of examining knowledge as of totally dissimilar views as well as to the point it into helpful info. Readily available methods are many foremost knowledge removal procedures so as to be urbanized as well as be employed within information processing comes that embody organization, categorization, grouping, In-order prototypes, and forecast as well as conclusion hierarchy. In the middle of diverse chores in knowledge processing, In-order prototype processing be the foremost vital chores. Successive example mining includes the mining of the subsequence's that appear extra minutes in an arrangement of groupings. It has a scope of uses in a few areas like the investigation of customer buy designs, protein grouping examination, DNA examination, quality succession examination, web access designs, and seismologic learning and climate perceptions. Different models and calculations have been produced for the sparing mining of sequent examples in awesome measure of data. In the proposed system, we recommends a new algorithm named "DATA because it IS Algorithm" to seek out frequent sequent patterns and prune away the infrequent things at the starting stages of the method. The experimental evaluation delineate that the projected platform algorithmic program performs effectively and effectively and outscores the existing algorithms by an order of magnitude.
\end{abstract}

Keywords: Sequential patterns, without ordering, frequent patterns $* * *$

\section{INTRODUCTION}

Consecutive example is an arrangement of thing sets organized in succession data-base' which happens successively with a particular request. An arrangement database' is an arrangement of requested components or occasions, put away with or without a solid idea of time. Everything set contains an arrangement of things which incorporate the same exchange time esteem. While affiliation rules show intra-exchange connections, successive examples speak to the relationship between's exchanges. Successive example processing is the procedure that concentrates certain consecutive examples whose backing surpasses a predefined negligible bolster limit. Moreover, successive example processing separates the groupings which mirror the most continuous practices in the arrangement data-base', which thus can be translated as area learning for a few purposes.

To diminish the gigantic numeral of' groupings keen on mainly intriguing progressive cases as well as to' get together the' various customer necessities, this-is crucial employ base reinforces which'-prunes' successive case by means of negative attention. Doubtlessly a higher sponsorship of a progressive illustration is favored for all the all the more interesting back to back cases. Back to back case mining is used as a part of a couple spaces. SPM is used as a piece of business relationship to study customer hones. Additionally, SPM is used as a piece of computational science to separate the amino destructive change plans. SPM is moreover used as a part of the scope of mesh employ removal to mine few mesh firewood scattered on various server-s'. Starting late, a couple of counts intended to S-P-M' contain future as well as mainly by far imperative as well as previous estimations rely top of upon possessions of the Apriori' figuring planned with Agrawal' as well as Sri-kant' in' 1994'.

This possession communicates so as to progressive illustration holds sub'-plans so as to be inside this way visit. Checking supposition, movement figurings have projected in' 1995', level outlining procedure shown in 1996' with similar past makers as well as Apriori'-based vertical masterminding system. All the additional starting late, summary counts in light of information projection have been-proposed.

Among' those' estimations: Free-Span planned by' H-a-n e-t a-1. In' 2000', Prefix-Span projected by' P-e-i' e-t a-l. In'2001', S-P-A-M (Apri-ori-bas-ed sure period as well as trimming) planned by' Ayr-es e-t a-1. In-2002'.Clo-Sp-an planned with Y-a-n e-t a-1. In-2003' as well as T-S-P planned with Tzv-et-kov e-t a-1. In-2005' a great segment of these figurings wo-ul-d' b-e clarified afterwards.

\subsection{Documentations-\&-Grouping of Prototypes}

Because communicated more than, course of action database' be a plan of asked for progressions. Gathering asked for summary of' proceedings, implied $<$ e1 e $2 \ldots$ el $>$. Given 
two game plans $\alpha=<\mathrm{x} 1 \mathrm{x} 2 \ldots \mathrm{xn}>$ and $\beta=<\mathrm{y} 1$ y $2 \ldots \mathrm{ym}>$, then $\alpha$ is known as, meant numbers $\mathrm{x} 1 \subseteq \mathrm{yj} 1, \mathrm{x} 2 \subseteq \mathrm{yj} 2, \ldots$, and $x n \subseteq y j n$. If $\alpha$ and $\beta$ have the going with courses of gathering catalogues' evidenced' in-to th-e' Table'-1', this is' probable' t'o discover the' absolute game plan customary sub-grouping.

Table 1: Succession' Data-base'

\begin{tabular}{|l|l|}
\hline SID & Sequence \\
\hline 10 & $\langle 1(\underline{\mathbf{l} \mathbf{m}})(\underline{\mathbf{l n}}) \mathrm{o}(\mathrm{nq})\rangle$ \\
\hline 20 & $\langle(\mathrm{lo}) \mathrm{n}(\mathrm{mn})(\mathrm{lp})\rangle$ \\
\hline 30 & $\langle(\mathrm{pq})(\underline{\mathbf{l}})(\mathrm{oq})(\underline{\mathbf{n} m})$ \\
\hline 40 & $\langle\mathrm{pr}(\mathrm{lq}) \mathrm{nmo}>$ \\
\hline
\end{tabular}

Each line in-to Table'-1 is a gathering. The progression have course of' action of segments. Every segment might hold a thing or'a' course of'action of things (set apart between fenced in area). Things inside a segment be un'ordered' as well as th-ey' recorded all together. As case, <1-(-m-n-)-o$n>$ be the subsequ-ence' of'- l'(-1-m-n')'. Rent exist the reinforce constrain slightest support identical to $2,\langle(\operatorname{lm}) n>$ is a true blue progressive case. A goliath figure of' likely progressive illustrations be concealed inside data-base's. In successive case removal issues, th-re-e' principal classes of cases can be communicated: Periodic or typical cases, truthful cases and vague cases [9]. We depict in the going with portions all the illustration classes.

\subsection{Irregular Prototypes}

These models's are' second-hand suspect an occasions of' a number of occasion (fused into the data'-set') later on as well as fathom inherent qualities consolidated into it. This model is from time to time too much restrictive, in light of the way that if' a little of' it-'s occasions be mis-aligned' i't' fa'ils' t-o' recognize a number of entrancing cases.

To' allow a predominant versatile replica', case be able mostly filled'. The' rule explanation behind existing in the direction of find the sub-sequen-ces' show schedule'in-the' data course of action. Case in point, stipulation to' contain' as' data progressions illustration discontinuous case since this is' repea-ted' with' a-peri-od' equivalent to' th-ree'. These past case are described filled discontinuous case in light of the way that every location case demonstrates a schedulings'. An' occupied irregular illustration be no-topen just a couple of utilizations, but instead various applications drive information progression anywhere no-teve-ry-pos-ition' demonstrates infrequent case. Case in point, if we have an information plan thusly case broad assortment things as well as readily available be negative filled incidental case by means of distance end to end 'three'. This is known as a-par-tial' irregular case.

\subsection{Verifiably Important Prototypes}

A mainly utilized way's worn to' assess dynamic cases be sponsorship as well as sureness. This'meas-ures be negative' basic intended to everyone livelihoods of' successive case mining. Fundamental or uncommon representations are the cases missed in the event that we' utilize th'e' gauge of' occasion-s (sorted out backing) a's-a'-meas-ure' significance. This issue analyzed by' different information removal applic-ation's'. Insi-de-two or three uses, clients are enamored by'-the' k-mos't' essential representations, as well as this errand can be effectively perceived utilizing an edge respect and the top $\mathrm{k}$ arranges that have a data grow more basic than the predefined edge ought to be returned. In any case, the issue data get worth is the weakness to see the scope events delineations.

Table 2: Compatibility matrix example

\begin{tabular}{|l|l|l|l|l|}
\hline \multirow{2}{*}{$\begin{array}{l}\text { Real } \\
\text { Values }\end{array}$} & \multicolumn{4}{|l|}{ Observed Values } \\
\cline { 2 - 5 } & $\mathbf{x}$ & $\mathbf{y}$ & $\mathbf{z}$ & $\mathbf{t}$ \\
\hline $\mathbf{x}$ & 0.9 & 0.12 & 0.0 & 0.05 \\
\hline $\mathbf{y}$ & 0.11 & 0.7 & 0.1 & 0.11 \\
\hline $\mathbf{z}$ & 0.0 & 0.0 & 0.88 & $0 . .12$ \\
\hline $\mathbf{t}$ & 0.1 & 0.17 & 0.0 & 0.78 \\
\hline
\end{tabular}

For example: if we have tow data outlines courses of action as takes after illustration get in the two progressions, scattered in-S-1-buts goes over successive i-n-S-2'. A couple mechanisms contain be obtainable within' [9']', intended to additional elucidation.

\subsection{Harsh Prototypes}

Boisterous itemsets or flawed information are min-ed' nearly as flawless itemsets. Flawed data happens in a couple of utilizations if the occasion of a case can't be seen. The two past delineated cases check simply revise competition of'the' case within-da-ta. An'inaccurate illustration be-a plan pictures which'happens with'a-val-ue more conspicuous tha'n-'an' inferred edge within-the-data' progression. In the direction of deal with issue of evaluated case divulgence, the makers possibility of calculability medium. These networks' give' a' probabilistic relationship from'-the' watched qualities t'o-the' certifiable qualities. These-calculability grid methodology proffer's-the' probability to'-figure certifiable sponsorship of'illustrations. Case in point, if we have a likeness matrix as in Table 2:

The watched thing $\mathrm{t}$ thinks about to a certified occasion, exclusively. An-importance-of' comparability system ought to be conceivable by the space expert, but there exist a couple of strategies to obtain the sensible estimation of each segment in the grid with a particular level of oversight.

\section{LITERATURE REVIEW}

The pioneer in this customary back to back case mining is Agarwal [1] who exhibited and handled the issue of progressive successive mining. For a given progressive data, the issue is to find each and every sequential case with a customer described slightest sponsorship, moreover named ceaseless back to back illustrations. In any case, Agarwal either considers sorted data or sorts the data before taking care of. A balanced Apriori' named Apriori'-All [1] was in like manner exhibited by Agarwal however these two counts 
doesn't discover plans without sorting the data. Different counts in light of the working standard of Apriori' were introduced by pros yet none was proposed to find the case of unsorted data without sorting the data. The Apriori'-like progressive illustration mining strategies encounter the evil impacts of the costs to handle a possibly enormous course of action of contender illustrations and breadth the data-base' again and again. The central injury of Apriori' based philosophy is voluminous confident time especially 2itemset candidates. The SPAM [2] computation uses bitmap representations to find the I-Extended groupings and Extended progressions yet SPAM estimation expect the dataset courses of action as a sorted one or it explicitly sorts the progressions before finding the successive cases. Continuous illustration mining counts using the vertical association are greatly capable, it can figure the sponsorship of candidate case by avoiding extravagant. Speedy Vertical Mining of Sequential Patterns[3]data-base' checks. Regardless, the major execution bottleneck of vertical mining figurings is that they generally contribute bundle of vitality evaluating contenders that don't appear in the data data-base' or are intermittent. This can be used for pruning candidates made by vertical mining figurings. An improved Apriori' figuring for connection rules [4] is proposed through reducing the time ate up in trades sifting for candidate thing sets by diminishing the amount of trades to be checked. At whatever point the $\mathrm{k}$ of $\mathrm{k}$-thing set forms, the opening between our upgraded Apriori' and the main Apriori' increases from viewpoint of time ate up, and at whatever point the estimation of minimum support extends, the cleft between our improved Apriori' and the principal Apriori' decreases from point of view of time consumed. The time used to make cheerful reinforce number in our improved Apriori' is not precisely the time ate up in the principal Apriori'.

Examination back to back illustrations mining[5,6] strategies, for instance, Apriori'-based estimations encounter the issue that various breadths of the data-base' are required remembering the final objective to make sense of which hopefuls are truly visit. Most by far of the plans gave so far to lessening the computational cost occurring due to the Apriori' property use a bitmap vertical representation of the passage progression data-base' and use bitwise operations to figure support at each accentuation. The changed vertical data-base's, in their turn, present overheads that lower the execution of the proposed estimation, however not as is normally done more terrible than that of illustration advancement figuring.

PROBLEM

\section{OBJECTIVE}

AND

\section{METHODOLOGY}

\subsection{Definition}

Let $\mathrm{I}=\{\mathrm{i} 1 ; \mathrm{i} 2 \ldots \ldots$.,in $\}$ be the game plan for goodness' sake. We consider an itemset as a subset of things. A gathering (event) $\mathrm{s}$ is set of itemsets and asked for it as demonstrated by time-stamp associated with them. The progression $\mathrm{s}$ is implied as s-1-,--s-2-,-.....,-s-1-, wheresj of $s$. S-j-i-smoreover call-ed-a segment gathering, as well as implied a thing. A thing can happen at most once in an itemset yet can display diverse times in various itemsets in a course of action. The amount of events of things in a progression is known as the gathering. The'-gathering with-in len-gth' l'is known as a l'sS.

\subsection{Issue-Statement}

Given a plan data-base' and as far as possible regard, the charge of progressive case removal be in the direction of discover the-whole course o-f' action of back to back case in the data-base'. Four systems specifically GSP, SPADE, PrefixSpan and FreeSpan are used for delivering courses of action and the execution of these estimations are penniless down and took a gander at for finding the as indicated by data mining speculation, successive case digging computation can be generally parceled into three social occasions: Apriori' based (GSP, SPADE, SPAM), outline advancement (FreeSpan, PrefixSpan) as showed up in Figure1.

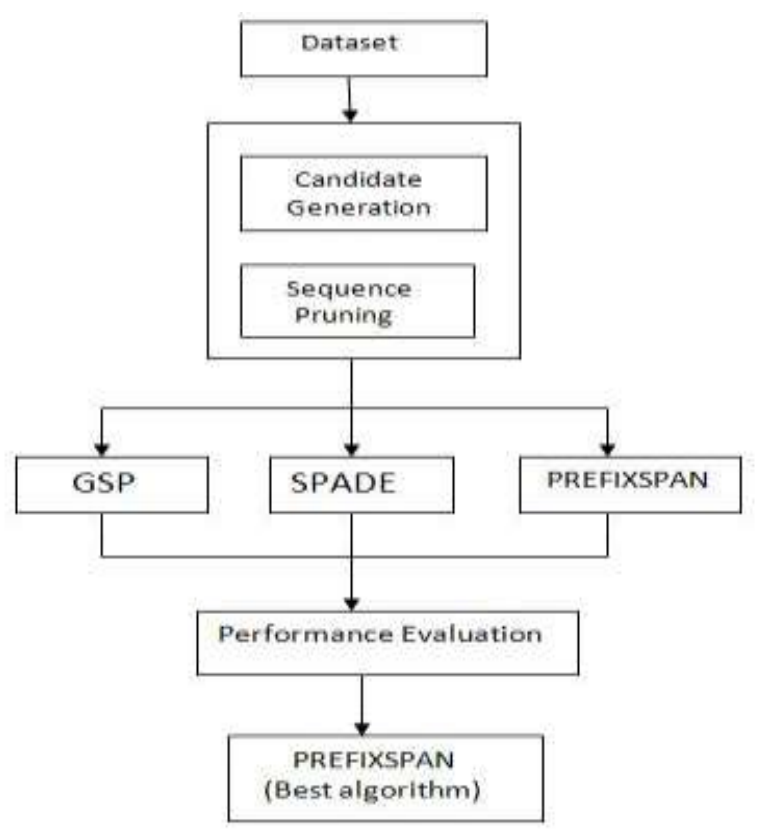

Fig.1 The system architecture

\section{PROPOSED ALGORITHMIC APPROACHES TO THE TASK OF SEQUENCE MINING}

Right when working up estimation to the attempt of movement mining, the thought is to make it more beneficial to the degree memory necessities and to lessen however much as could be typical the reaction time [10]. That will propose the use of appropriated information structures and the utilization of old and novel algorithmic ways to deal with oversee play out this attempt.

The estimation contains different methodology and the systems are perceived here,

Procedure InitialProcess( Dataset D, min-sup V) Input: Dataset D

OutPut: Normalized Converted Data 
Begin:

1. Load and Scan the Dataset D

2. "DatarowDrî D do Mark UniqueItems in InitialTable

Mark Numerical Value for UniqueItems in InitialTable Mark Items in ItemLocation Table

Find NumberOfEventsnEÎDr

Index the Item numerical values to ItemLocation Table IF $[n E=1]$ Mark 0

Else Mark Numerical Values separated by comma End IF

End For

3. Mark Count of UniqueItems, IF [ count <V] Prune UniqueItem

End Procedure

Procedure FindProbableCombinations(InitialTableInT)

Input: InitialTableInT

Output: Probable combinations

Begin:

Find Thetotalitems Tot in InT

"Index I ÎTot

"Index $I+1 \hat{I}$ Tot

ConcateInT[I],InT[I+1 $]^{\grave{a}}$ Combination

End For

End For

Return Combination

End procedure

Procedure Data-as-it-IS( Dataset D, min-sup $\square$ )

Input: Dataset D, minimum support $\square$

Output: Frequent Sequential patterns

Begin:

Call InitialProcess $(D, \square)$

Call FindProbableCombinations $(T)$

$\square$ Combination $C \square$ Combination do

Loop Until (Sequences in $C \neq \square$ )

Load Sequence from ItemLocationTable according to $C$ Check for a non-empty value in the first Column

IF Row[Column1] = Non-empty, Start from that row

Concatenate the non-empty value by moving UP,Right, Down, Diagonal IF (Items found in same Column)

Check the EventIndex table to find the numerical values Concatenate according to numerical values as a single sequence End if

Store the patterns ${ }^{\square}$ temp End if

End Loop
Count the patterns and check $\square \square \quad$ Store frequent sequentialpatterns Else

Ignore infrequent patterns End For

Return Frequent sequential patterns End Procedure

The proposed algorithm DAIS is the way to discover the frequent sequential patterns without ordering the items. At first the column 1 is checked for a non-empty value. If the non-empty value found, the process starts from that row. Move from the first column towards right, up or down to identify a non-empty value. After identify the non-empty value, all the items were concatenate and form the sequential patterns. This method employs event item indexing for each sequence and uses numerical values for events which contains more than one item. This methodology efficiently finds and discovers the frequent sequential patterns without disturbing the data present in the events of a sequence.

This algorithm proposed a new procedure to find a probable combination of unique items. Step 1 finds the total number of unique item to identify the combination. Step 2 identify the index value to locate the items. In Step 3 the Item combinations are found to check whether the combinations are higher than the minimum support value provided by the user. The result is the combination of items produce a patterns. From the table 3, the data gathering data-base' $\mathrm{S}$ is a course of action of tuples (sid, s), where sid is the progression identifier and $\mathrm{s}$ is the data gathering. The amount of tuples in S data-base' is called base size of the data-base' $S$, and demonstrated in gathering Sa, Sais-s. The sponsorship of a plan Sain the data-base' $S$ be the'amount of tup-le-s during th-e-data-ba'se' enclosing Sa, implied as $\sup (\mathrm{Sa})$.

For a given positive entire number m-in'-sup, a's-t'he' hold up edge, the' progression Sais call-e-d-const'ant-seq-uential' case in data-base' $S$, if $\sup (\mathrm{Sa}) \square$ min'-su-p'. By and large the case is intermittent.

Table 3: Sample data-base' of unordered events

\begin{tabular}{|l|l|}
\hline SeqID & Sequences \\
\hline S1 & {$[$ a $($ dc $)$ ad $]$} \\
\hline S2 & {$[$ acae $]$} \\
\hline S3 & {$[$ cad $($ cbd $)]$} \\
\hline S4 & {$[$ bbc $]$} \\
\hline S5 & {$[$ (bcd)d $]$} \\
\hline
\end{tabular}

The proposed approach primaries scrutinize the record-base in the direction of discovering the unique points present into the record-base. A table is constructed to identify whether the item is present in the sequence and if the item is present then it is denoted by " 1 " else denoted by " 0 ". Along with this the support count of every unique item is calculated and stored. A numerical value for the items are provided and marked in this table as shown in the table 4. 
Let us consider $\mathrm{S} 1=[\mathrm{a}(\mathrm{dc}) \mathrm{ad}]$, here there are four sequences and for brevity, the brackets are omitted and [(a) (dc) (a) (d)] is written as [a (dc) ad]. The sequence S1 contains four events and the second event $(\mathrm{dc})$ is unsorted and this event or subsequence is not sorted to discover the recurrent chronological prototypes in the proposed approach.

Table 4: Identification of unique items with count and numerical value

\begin{tabular}{|l|l|l|l|l|l|l|}
\hline S1 & S2 & S3 & S4 & S5 & $\begin{array}{l}\text { SUPPORT } \\
\text { COUNT }\end{array}$ & $\begin{array}{l}\text { VUMERIC } \\
\text { VALUE }\end{array}$ \\
\hline 1 & 1 & 1 & 0 & 0 & 3 & 1 \\
\hline 0 & 0 & 1 & 1 & 1 & 3 & 2 \\
\hline 1 & 1 & 1 & 1 & 1 & 5 & 3 \\
\hline 1 & 0 & 1 & 0 & 1 & 3 & 4 \\
\hline 0 & 1 & 0 & 0 & 0 & 1 & 5 \\
\hline
\end{tabular}

After finding the unique items in the data-base' the following table is constructed initially to recognize whether the sequences contain a particular item or not.

\section{ITEM LOCATION AND EVENT INDEXING}

Now the unique items exact location in each sequence is found and marked with the item name or simply that location is left empty. Along with this marking of location, the number of items in every event is found and the numerical values corresponding to the items be stocked up into the table-chart like exposed in the figure 2.

Table 5: Locating items table for sequence S1, S2, S3, S4, S5

\begin{tabular}{|l|l|l|l|l|l|l|l|}
\hline S & \multicolumn{9}{|l|}{ Sequence } & \multicolumn{4}{l|}{ Event } \\
\hline \multirow{4}{*}{ S1 } & E1 & a & $*$ & $*$ & - & 1 & 0 \\
\cline { 2 - 8 } & E2 & $*$ & c & d & - & 2 & 4,3 \\
\cline { 2 - 8 } & E3 & a & $*$ & $*$ & - & 1 & 0 \\
\cline { 2 - 8 } & E4 & $*$ & $*$ & d & - & 1 & 0 \\
\hline \multirow{4}{*}{ S2 } & E1 & a & $*$ & $*$ & - & 1 & 0 \\
\cline { 2 - 8 } & E2 & $*$ & c & $*$ & - & 1 & 0 \\
\cline { 2 - 8 } & E3 & A & $*$ & $*$ & - & 1 & 0 \\
\cline { 2 - 8 } & E4 & $*$ & $*$ & e & - & 1 & 0 \\
\hline \multirow{5}{*}{ S3 } & E1 & $*$ & $*$ & c & $*$ & 1 & 0 \\
\cline { 2 - 8 } & E2 & a & $*$ & $*$ & $*$ & .3 & 1.0 \\
\cline { 2 - 8 } & E3 & $*$ & $*$ & $*$ & d'1 & 3.1 & 2.0 \\
\cline { 2 - 8 } & E2 & b & $*$ & - & - & 1 & 0 \\
\cline { 2 - 8 } & E3 & $*$ & c & - & - & 1 & 0 \\
\cline { 2 - 7 } & E2 & $*$ & $*$ & d & - & $2,3,4$ & 0 \\
\hline \multirow{5}{*}{} & & & & & \\
\end{tabular}

Here in figure 2, the sequence $\mathrm{S} 1$ is considered and $\mathrm{S} 1$ consists of 4 events [E1,E2,E3,E4] and the unique items in this sequence is identified and located in the corresponding events as shown in the above figure 1. Similarly number of items in each event is found and if it found value exceeds 1 , the corresponding item's numerical value is stored as shown. Here in event indexing 4 denotes "d" and 3 denotes "c".

\section{FORMATION OF ITEM COMBINATIONS}

After locating the items in the sequence, the item combinations are found to check whether the combinations are higher than smallest amount hold up value provided by the user assuming mun-sup value is 2 .

First item "a" is considered and the probable combinations are formed for the entire unique items. Here since " $\mathrm{e}$ " item hold up tally is less than the least amount carry rate, it is eliminated. To check whether the combination items produce patterns, AND operation is performed as shown below.

Table 6: Combination formation of " $a b, b c, b c d, a b c$, acd" to generate frequent patterns

\begin{tabular}{|l|l|l|l|l|l|}
\hline Item & S1 & S2 & S3 & S4 & S5 \\
\hline bcd & 0 & 0 & 1 & 0 & 1 \\
\hline abc & 0 & 0 & 1 & 0 & 0 \\
\hline acd & 1 & 0 & 1 & 0 & 0 \\
\hline
\end{tabular}

Since "ab" support count is 1 , the corresponding item sets which contains "ab" will also be 1 . So the entire "ab" combinations are eliminated and pruned away. Combination formation of "ac" to generate frequent patterns. Since "e" is eliminated at the first stage, the 4-itemset combination is not considered. Similarly all the unique item combinations are formed and checked whether the count is higher or equal to the minimum support value provided. Those combinations which are leading or equivalent to the lowest amount carry values are further processed top discover the frequent sequential patterns.

\section{EXPERIMENTAL RESULTS}

The proposed DAIS algorithm was implemented Microsoft C\#.NET programming language resting up a system by means of $2.66 \mathrm{GHz}$ Intel-Pentium core2duo processor with 1GB RAM running on windows 7 ultimate. The evaluations are carried out on artificial information produced with I-B$\mathrm{M}$ artificial marketplace hamper information producer. An input'ed strictures used for comparison are given below in the Table 8.

Table 7: Dataset parameters

\begin{tabular}{|l|l|l|l|}
\hline Parameters & Description of parameter & Dataset utilized for evaluation \\
\hline D & No. of successions in thedataset & $3 \mathrm{~K}$ & $12 \mathrm{~K}$ \\
\hline C & standard events for each succession & 5 & 12 \\
\hline S & Average length of potentiallyfrequent sequential patterns & 5 & 8 \\
\hline I & Average length of item sets inMaximal potentially frequentpatterns & 5 & 8 \\
\hline
\end{tabular}


The evaluation is done using different minimum support values and varying number of total sequences in the dataset, the proposed algorithm clearly outscores Apriori'-All and matches nearly with the execution speed of SPAM. The proposed algorithm is executed on small, medium and large datasets and it clearly performs better on most of the situations with respect to speed and memory space. The DAIS and SPAM algorithm performed well in large dataset due to the recursive steps it performs during pattern finding.
The proposed algorithm performed quite well on small and medium datasets when compared to its counterparts. As far as the memory footprints are concerned, SPAM performed reasonably better than the proposed algorithm mainly due to the bitmap representation of data. A numeral amount of frequent chronological prototypes found in-the proposed algorithms are definitely lower than-that of the existing algorithm since the proposed algorithm never sorts the sub sequences.

Table 8: Evaluated results with respect to execution time

\begin{tabular}{|c|c|c|c|c|c|c|}
\hline Dataset & \multicolumn{3}{|c|}{ D5KC5T5S5I5 } & \multicolumn{3}{|c|}{ D12KC12T12S8I8 } \\
\hline \multirow{3}{*}{ Algorithm } & \multicolumn{3}{|c|}{ ( Synthetic Data set) } & \multicolumn{3}{|c|}{ ( Synthetic Data set) } \\
\hline & \multicolumn{3}{|c|}{ Minimum Support values } & \multicolumn{3}{|c|}{ Minimum Support values } \\
\hline & 2 & 3 & 4 & 5 & 6 & 7 \\
\hline Apriori'-All & 86 & 71 & 63 & 112 & 103 & 81 \\
\hline SPAM & 58 & 49 & 28 & 37 & 29.2 & 23 \\
\hline DAIS & 60 & 51 & 31 & 38 & 28 & 23.32 \\
\hline
\end{tabular}

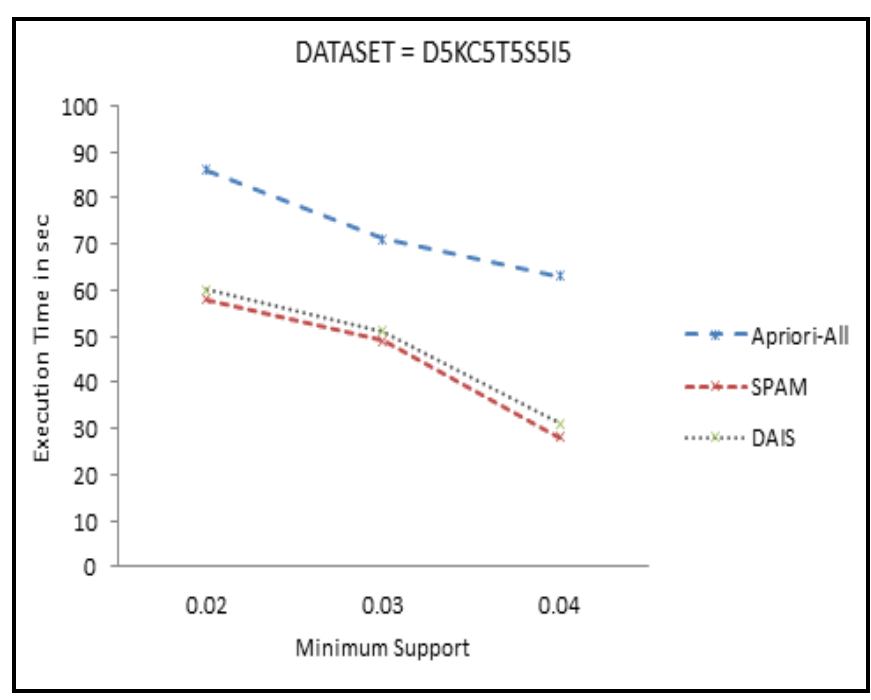

Fig 2(a): Execution time Evaluation

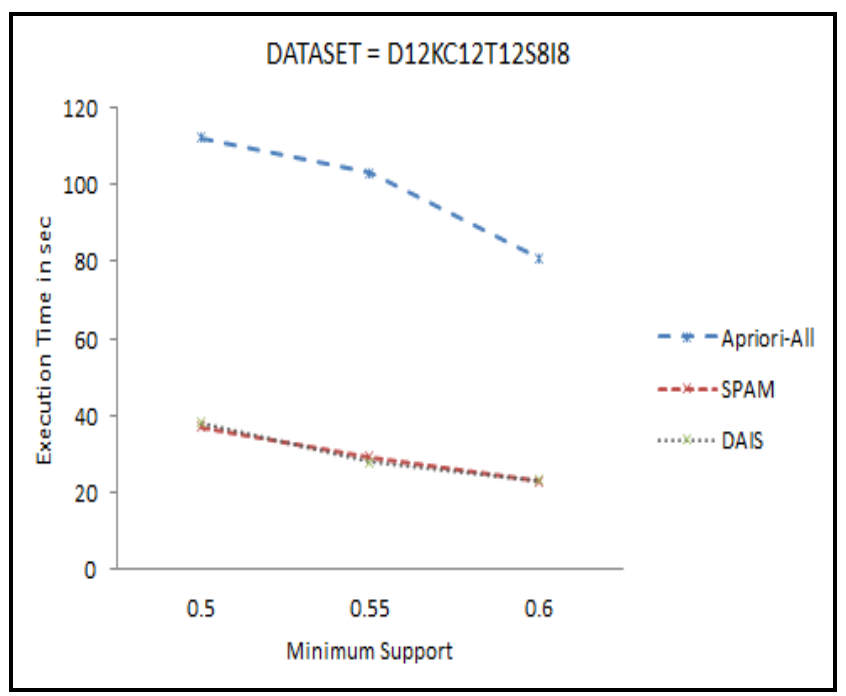

Fig 2(b): Execution time Evaluation

From the Table 8 and from the figure 2(a),2(b) it is quite clear that the proposed algorithm DAIS performed well when compared to Apriori'-All and matched the performance of SPAM. SPAM algorithm performance is better for small dataset, and DAIS performed extremely well for large dataset.

Table 9: Evaluation results with respect to memory consumption

\begin{tabular}{|c|c|c|c|c|c|c|}
\hline Dataset & $\begin{array}{l}\text { D5I } \\
\text { ( Sy }\end{array}$ & $\begin{array}{l}\mathrm{T5S5} \\
\text { ic Da }\end{array}$ & & $\begin{array}{l}\text { D12 } \\
\text { ( Sy }\end{array}$ & $\begin{array}{l}\text { 2S8I8 } \\
\text { ata se }\end{array}$ & \\
\hline \multirow{2}{*}{ Algorithm } & \multicolumn{3}{|c|}{ Minimum Support values } & \multicolumn{3}{|c|}{ Minimum Support values } \\
\hline & 0.1 & 0.15 & 0.2 & 0.1 & 0.15 & 0.2 \\
\hline Apriori'-All & 209 & 185 & 153 & 359 & 331 & 311 \\
\hline SPAM & 161 & 147 & 121 & 287 & 253 & 221 \\
\hline DAIS & 163 & 146 & 119 & 279 & 245 & 209 \\
\hline
\end{tabular}




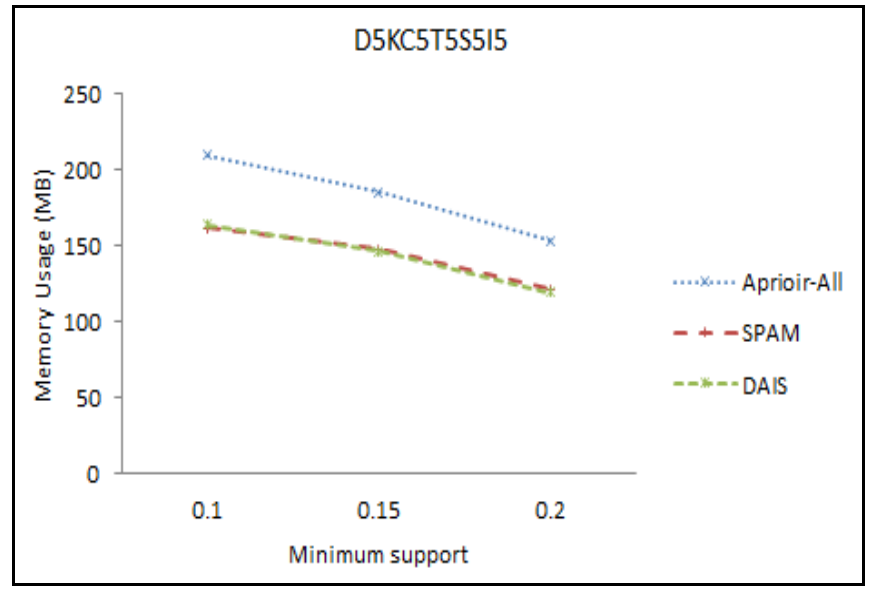

Fig 3(a): Execution memory usage

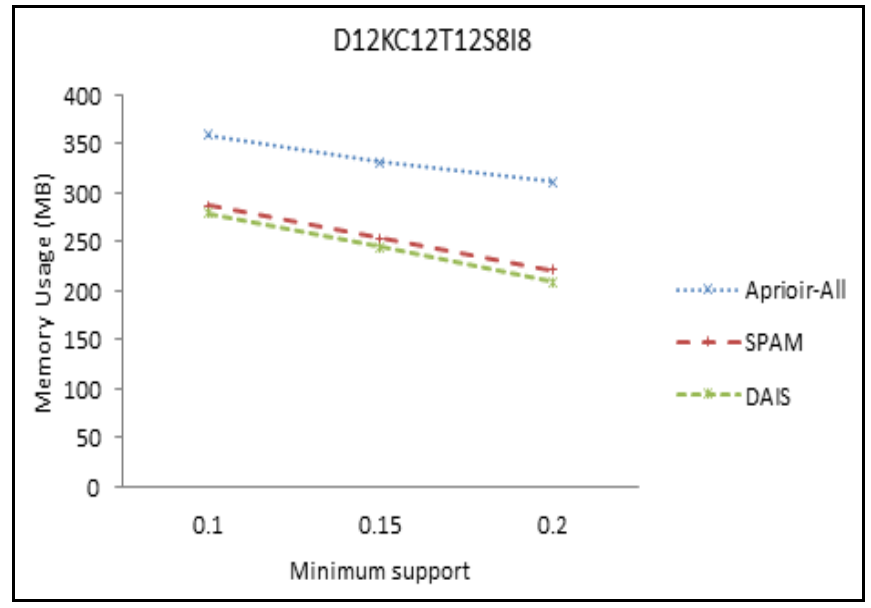

Fig 3 (b): Execution memory usage

From the table 9 and from the figure 3(a),3(b) it is quite clear that the memory usage of the proposed algorithm was compared with the existing algorithms and from the evaluated results, it is quite clear that the DAIS algorithm performed exceedingly well on larger datasets and at the same time the SPAM performed well on both small and larger dataset mainly because of the bitmap representation used in SPAM.

\section{CONCLUSION}

Back to back mining has been attracting thought in late research in the field of data mining. Since the interest space is gigantic and data volume is tremendous, it has made various issues for mining back to back cases. To reasonably mine the continuous illustrations, capable progressive case mining computations are required. Among the back to back case counts GS-P, SPA-DE and Prefix-Span, Prefix-Span, DAIS is a capable illustration improvement technique since it beats the other three computations. Clearly DAIS Algorithm is more capable with respect to running time, space use and adaptability than Apriori' based computations. Another estimation to meet the test of discovering progressive case without asking for the events in a gathering is proposed in this paper and the proposed count DAIS performed growing awesome for greater datasets without sorting by using event thing indexing using numerical qualities. The proposed figuring should be executed on denser datasets with little min-sup qualities to test the adequacy and the accuracy.

\section{REFERENCES}

[1]. R. Agarwal and R. Srikant. Mining Sequential Pattern, Fast Algorithm for Mining Association Rule in Large Database's.In Proc.1995,1994 Int. Conf. Data Engineering, pages 3-10, 1995., Int. Conf. Very Large Data-base's, pp. 487-499, 1994.

[2]. J. Ayres, J. Flannick, J. Gehrke, and T. Yiu. Sequential PAttern Mining Using A Bitmap Representation. In Proc. 2002 Int. Conf. Knowledge Discovery and Data Mining, 2002.

[3]. Philippe Fournier-Viger1, Antonio Gomariz2,Manuel Campos2, and Rincy Thomas3: In Proc.2014 Fast Vertical Mining of Sequential Patterns Using Co-occurrence Information,University de Moncton

[4]. Mohammed Al-Maolegi1: In Proc.2014 An improved Apriori' algorithm for association rules. International Journal on Natural Language Computing (IJNLC) Vol. 3.

[5]. Nikhil Gundawar1, Venkatesh Akolekar2, Piyush Phalak3, Akshay Gujar4 and Bewoor5: In Proc.2014 Analysis of Sequential Pattern Mining: international journal for research in emerging science and technology, volume-1, issue-6.

[6]. Bhawna Mallick, Deepak Garg and Preetam Singh Grover. Constraint-Based Sequential Pattern Mining: In Proc. 2014 The International Arab Journal of Information Technology, Vol. 11, No. 1, January 2014.

[7]. Goswami, Chaturvedi Anshu, Raghuvanshi: In Proc.2010 An Algorithm for Frequent Pattern Mining Based On Apriori'. International Journal on Computer Science and Eng

[9]. Ms Shweta, Dr. Kanwal Garg2:In Proc.2013Mining Efficient Association Rules Through Apriori' Algorithm Using Attributes and Comparative Analysis of Various Association Rule Algorithms. International journal of advanced research in computer science and software engineering. 